\title{
THE GENERALIZED GAMMA FUNCTION, NEW HARDY SPACES, AND REPRESENTATIONS OF HOLOMORPHIC TYPE FOR THE CONFORMAL GROUP ${ }^{1}$
}

\author{
BY KENNETH I. GROSS, WAYNE J. HOLMAN, III AND RAY A. KUNZE
}

Communicated by J. A. Wolf, January 3, 1977

Operator valued generalizations of the integral formula for the classical gamma function arise in connection with noncompact semisimple, or reductive, Lie groups for which the symmetric space $G / K$ is Hermitian, and they relate to various problems in analysis, group representations, and number theory. In particular, when the holomorphic discrete series for $G$, constructed originally by Harish-Chandra [3], is realized in terms of the unbounded form of $G / K$ as a Siegel domain, the gamma function plays a decisive role (cf., [1], [2a], [2b], [2d], [6a] , [6b]). Moreover, the holomorphic discrete series has an analytic continuation [7], the full extent of which is controlled by the analytic continuation of a normalized version of the gamma function. In general, however, it is only when the gamma function is scalar valued, an occurrence which accounts for but a small part of the holomorphic discrete series, that the full analytic continuation has been determined. In that specialized context, it is known from [6b] that Hardy type Hilbert spaces associated to the various boundary components of $G / K$ appear at the "integer points" in the analytic continuation.

This note announces rather complete solutions to these problems for the conformal group $G=U(2,2)$. Specifically, we give the entire analytic continuation of the gamma function, the full extent of analytic continuation of the holomorphic discrete series, and we introduce some new vector-valued Hardy spaces.

I. The generalized gamma function. Let $\mathrm{A}=A \times A$ where $A=\mathrm{GL}(2, \mathrm{C})$ and fix a complete set of irreducible holomorphic finite-dimensional representations $\lambda$ of $A$ such that $\lambda\left(a_{1}, a_{2}\right)^{*}=\lambda\left(a_{1}^{*}, a_{2}^{*}\right)$. Let $\lambda$ be parametrized by a pair of highest weights $\left(\sigma_{j}+2 l_{j}, \sigma_{j}\right),(j=1,2)$, where $\sigma_{j}$ and $2 l_{j}$ are integers and $l_{j} \geqslant 0$. Then $\lambda=\lambda\left(\cdot ; \sigma_{1}, \sigma_{2}, \lambda^{0}\right)$ where

$$
\lambda\left(a_{1}, a_{2}\right)=\Delta\left(a_{1}\right)^{\sigma}{ }_{1}\left(a_{2}\right)^{\sigma} \lambda^{0}\left(a_{1}, a_{2}\right)
$$

with $\Delta=\operatorname{det}$ and $\lambda^{0}=\lambda^{0}\left(\cdot ; l_{1}, l_{2}\right)$ a polynomial representation. Let $P$ be

AMS (MOS) subject classifications (1970). Primary 22E30, 22E45, 33A75, 43A80; Secondary 32M15, 33A15, 81 A78, 46E20.

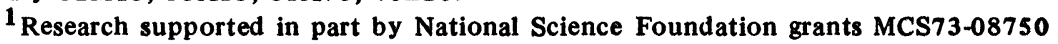
and GP-36565X. 
the cone of positive matrices in $A$. The generalized gamma function for $G$ of weight $\lambda^{0}$ is the holomorphic operator-valued function defined by the absolutely convergent integral

$$
\Gamma_{\lambda 0}(\alpha)=\int_{P} e^{-\operatorname{tr}(r)} \lambda^{0}(r, \bar{r}) \Delta(r)^{\alpha-2} d r
$$

for $\operatorname{Re}(\alpha)>1$ and elsewhere by analytic continuation.

The following is the main technical theorem. It is proven from the Clebsch-Gordon formula by an involved calculation.

THEOREM 1. Fix $\lambda^{0}$. The values $\Gamma_{\lambda 0}(\alpha)$ for $\operatorname{Re}(\alpha)>1$ form a commutative family of normal operators having distinct eigenvalues

$$
\gamma_{l}^{\lambda 0}(\alpha)=\frac{\Gamma\left(\alpha+2 l_{1}\right) \Gamma\left(\alpha+2 l_{2}\right) \Gamma\left(\alpha+2 l_{1}+2 l_{2}+1\right) \Gamma(\alpha-1)}{\Gamma\left(\alpha+l_{1}+l_{2}-l\right) \Gamma\left(\alpha+l_{1}+l_{2}+l+1\right)}
$$

indexed by $l=\left|l_{1}-l_{2}\right|,\left|l_{1}-l_{2}\right|+1, \ldots, l_{1}+l_{2}$.

COROLlaRY 1. The function $\alpha \rightarrow \Gamma_{\lambda} 0(\alpha)^{-1}$ extends from $\operatorname{Re}(\alpha)>1$ to an entire function of $\alpha$.

We remark that in the special case $l_{1}=0$ or $l_{2}=0$ (cf., [2a]), $\Gamma_{\lambda} 0$ is scalar-valued.

Let $t_{\lambda 0}(\alpha)=\operatorname{tr}\left(\Gamma_{\lambda 0}(\alpha-2)^{-1} \Gamma_{\lambda 0}(\alpha)\right)$, and define the normalized gamma function $N_{\lambda 0}$ by $N_{\lambda 0}(\alpha)=t_{\lambda 0}(\alpha) \Gamma_{\lambda 0}(\alpha-2)$.

Corollary 2. (i) The function $\alpha \rightarrow N_{\lambda}(\alpha)^{-1}$ is entire.

(ii) If either $l_{1}=0$ or $l_{2}=0$ (so $N_{\lambda 0}(\alpha)$ is scalar), then $N_{\lambda 0}(\alpha)^{-1}$ is positive for $\alpha>1$, and $N_{\lambda} 0(1)^{-1}=0$.

(iii) If both $l_{1} \neq 0$ and $l_{2} \neq 0$, then $N_{\lambda}(\alpha)^{-1}$ is a positive operator for $\alpha>2$, and $N_{\lambda} 0(2)^{-1}$ is nonnegative (one eigenvalue is positive and all others vanish).

Let $P_{1}=\left\{r \in C^{2 \times 2}: r=r^{*} \geqslant 0\right.$ and $\left.\Delta(r)=0\right\}$, the rank one boundary component of $P$, and set

$$
\tilde{\Gamma}\left(\lambda^{0}\right)=\int_{P_{1}} e^{-\operatorname{tr}(r) \lambda^{0}(r, \bar{r}) d m(r)}
$$

where $m$ is relatively $A$-invariant measure on $P_{1}$.

COROllary 3. The operator $\widetilde{\Gamma}\left(\lambda^{0}\right)$ is positive. In fact, $\widetilde{\Gamma}\left(\lambda^{0}\right)=$ $\lim _{\alpha \rightarrow 1}\left(\Gamma_{\lambda} 0(\alpha) / \Gamma(\alpha-1)\right)=c\left(\lambda^{0}\right) N_{\lambda} 0(3)$, where $c\left(\lambda^{0}\right)$ is real and positive.

II. Analytic continuation of the holomorphic discrete series. We realize $G / K$ as the Siegel upper half plane $H=S+i P$ in $C^{2 \times 2}$ where $S=\left\{x \in C^{2 \times 2}\right.$ : $\left.x=x^{*}\right\}$. The relative holomorphic discrete series for the universal covering group $\widetilde{G}$ of $G$ consists of representations $T(\cdot, \lambda)$ indexed by $\lambda$ as in (1) with $\sigma_{1}, \sigma_{2}$ real and $\alpha=\sigma_{1}+\sigma_{2}>3 . T(\cdot, \lambda)$ acts in the space $H\left(\lambda^{0}, \alpha\right)$ of 
holomorphic vector-valued functions $F$ such that

$$
\|F\|_{\lambda 0, \alpha}^{2}=\int_{H}\left\|\lambda^{0}(y, \bar{y})^{1 / 2} F(x+i y)\right\|^{2} \Delta(y)^{\alpha-4} d x d y<\infty .
$$

When $\sigma_{1}$ and $\sigma_{2}$ are integers, $T(\cdot, \lambda)$ is a representation of $G$ itself. $H\left(\lambda^{0}, \alpha\right)$ $\neq 0$ if and only if $\alpha>3$. The reproducing kernel $Q_{\lambda, \alpha}$ for $H\left(\lambda^{0}, \alpha\right)$ is calculated (cf., [2d]) to be

$$
Q_{\lambda 0, \alpha}(z, w)=\int_{P} e^{j \operatorname{tr}\left(z-w^{*}\right) r} \lambda^{0}(r, r)^{1 / 2} N_{\lambda 0}(\alpha)^{-1} \lambda^{0}(r, r)^{1 / 2} \Delta(r)^{\alpha-2} d r
$$

for $z, w \in H$, which can be evaluated as

$$
Q_{\lambda 0, \alpha}(z, w)=\Delta\left(-i\left(z-w^{*}\right)\right)^{-\alpha} \lambda^{0}\left(-i\left(z-w^{*}\right),-i\left(z-w^{*}\right)^{t}\right)^{-1} .
$$

Following [6b], we define the Wallach set for $G$ to consist of all $\left(\lambda^{0}, \alpha\right)$ such that $Q_{\lambda 0, \alpha}$ given by (3) is positive-definite.

THEOREM 2. Fix any $\lambda^{0}$. Then: (i) If $l_{1}=0$ or $l_{2}=0$ (so $N_{\lambda 0}(\alpha)$ is scalar), then $\left(\lambda^{0}, \alpha\right)$ is in the Wallach set for $\alpha>1$. (ii) If $l_{1} \neq 0$ and $l_{2} \neq 0$, then $\left(\lambda^{0}, \alpha\right)$ is in the Wallach set if $\alpha \geqslant 2$.

The proof is immediate from (2) and Corollary 2. In Case (i), $N_{\lambda 0}(\alpha)^{-1}$ becomes negative for $\alpha<1$; and in Case (ii), $N_{\lambda 0}(\alpha)^{-1}$ ceases to be a nonnegative operator for $\alpha<2$. Therefore, Theorem 2 gives the complete analytic continuation of the holomorphic discrete series. The special case $\lambda^{0} \equiv 1$ (for generic $G$ ) is due to Rossi and Vergne [6b] , and Case (i) appears in [2a] and [4]. Case (ii) with $2 \leqslant \alpha<3$ yields new Hilbert spaces $H\left(\lambda^{0}, \alpha\right)$ and new irreducible representations of $\widetilde{G}$. The parameter $\alpha=3$, corresponds to a space $H\left(\lambda^{0}, 3\right)$ which realizes the limit of holomorphic discrete series in the sense of [5].

III. New Hardy spaces. Let $V\left(\lambda^{0}\right)$ be the space of $\lambda^{0}$ and denote by $\$^{2}\left(\lambda^{0}, P_{1}\right)$ the Hilbert space of all holomorphic $F: H \rightarrow V\left(\lambda^{0}\right)$ such that

$$
\|F\|_{\lambda 0, P_{1}}=\sup _{t \in P} \int_{S+i P_{1}}\left\|\lambda^{0}(y, \bar{y})^{1 / 2} F(x+i(y+t))\right\|^{2} d x d m(y)<\infty .
$$

TheOREm 3. $\mathfrak{Q}^{2}\left(\lambda_{0}, P_{1}\right)=H\left(\lambda^{0}, 3\right)$. In particular, $\mathfrak{S}^{2}\left(\lambda_{0}, P_{1}\right) \neq 0$.

The proof follows from Corollary 3 by a Plancherel theorem argument. For $\lambda^{0}=1$, Theorem 3 is given in [6b]. There, it is also shown that discrete points appear in the Wallach set (for $\lambda^{0} \equiv 1$ ) beyond the range of analytic continuation. The analytic implementation of such spaces for generic $\lambda^{0}$ is an interesting open problem.

\section{REFERENCES}

1. R. Godement, Fonctions automorphes, Séminaire Henri Cartan, 10e année: 1957/58, Secretariat Mathématique, Paris, 1958. MR 21 \#2750. 
2(a). K. I. Gross and R. A. Kunze, Fourier Bessel transforms and holomorphic discrete series, Conference on Harmonic Analysis, Lecture Notes in Math., vol. 266, SpringerVerlag, Berlin and New York, 1972, pp. 79-122. MR 51-6285.

2(b). - Generalized Bessel transforms and unitary representations, Harmonic Analysis on Homogeneous Spaces, Proc. Sympos. Pure Math., vol. 26, Amer. Math. Soc., Providence, R. I., 1973, pp. 347-350. MR 49 \#120.

2(c). - Bessel functions and representation theory. I, J. Functional Analysis 22 (1976), 73-105.

(to appear).

2(d). - Bessel functions and representation theory. II, J. Functional Analysis.

3. Harish-Chandra, Representations of semi-simple Lie groups. IV, V, VI, Amer. J. Math. 77 (1955), 743-777; ibid. 78 (1956), 1-41; ibid. 78 (1956), 564-628. MR 17, $282 ; 18,490$.

4. H. Jakobson and M. Vergne, Wave and Dirac operators, and representations of the conformal group, 1976 (preprint).

5. A. Knapp and K. Okamoto, Limits of holomorphic discrete series, J. Functional Analysis 9(1972), 375-409. MR 45 \#8774.

6(a). H. Rossi and M. Vergne, Representations of certain solvable Lie groups on Hilbert spaces of holomorphic functions and the application to the holomorphic discrete series of a semi-simple Lie group, J. Functional Analysis 13 (1973), 324-389.

6(b). - Analytic continuation of the holomorphic discrete series of a semisimple Lie group, Acta Math. 136 (1976), 1-59.

7. N. Wallach, Analytic continuation of the discrete series. I, II, Trans. Amer. Math. Soc. (to appear).

DEPARTMENT OF MATHEMATICS, UNIVERSITY OF NORTH CAROLINA, CHAPEL HILL, NORTH CAROLINA 27514

DEPARTMENT OF MATHEMATICS, UNIVERSITY OF THE SOUTH, SEWANEE, TENNESSEE 37375

DEPARTMENT OF MATHEMATICS, UNIVERSITY OF CALIFORNIA, IRVINE, CALIFORNIA 92664

BULLETIN OF THE

AMERICAN MATHEMATICAL SOCIETY

Volume 83, Number 3, May 1977

\section{ERRATUM, VOLUME 82}

Robert Lee Moore, 1882-1974, by R. L. Wilder

In Volume 82, p. 421 , line 14 should read: "analyzes both the form of the group of motions, and the underlying space". 